\title{
openaccess
}

\section{Barriers to VR use in HE}

\author{
Leighton Evans $₫ 0000-0002-6875-6301$ \\ Swansea University. Corresponding author: I.evans@swansea.ac.uk
}

VR promises revolutionary changes in the levels of immersion that users can experience, and if applied successfully in educational contexts this deep immersion could have significant effects on both teaching and learning. To utilise VR effectively in the higher education (HE) space, there must be some consideration given to what might prevent the use of VR in this sector and why these barriers exist-and how they can be mitigated against. Based on an extensive research project involving qualitative interviews with 21 VR makers and designers in autumn 2017 , following a thematic analysis of the interview data, this paper identifies 5 major barriers to the uptake of VR in a wider cultural sense and in a specific, educational context. These identified barriers are: the materiality of VR and issues with headsets and cables; interfaces within VR and issues with haptic technology; the 'language of VR' and the difficulty in communicating the benefits of VR; cybersickness and gender issues with VR use, and, the cost of VR. The preparation of educational VR materials requires an acknowledgement of these sometimes-concealed barriers to VR use, and it is proposed that through knowledge-transfer and sharing of best practice the use of VR in higher education could become a model of best practice for designing inclusive VR experiences that avoid major barriers to participation in VR.

\section{Introduction}

Imagine, as a history student interested in the Second World War, being able to experience being part of a Lancaster Bomber crew flying over Germany on the way to Berlin, rather than just reading second-hand accounts of what this experience was like; a kind of embodied time-travel back to a historically-significant set of events that could improve empathy and understanding of the conditions and context of such actions. Impossible? Yes and no. Thanks to the ' 1943 Berlin Blitz' VR experience (BBC, 2018) history students are now afforded the possibility of an embodied learning experience on a Lancaster Bomber, in the place of BBC war reporter WynfordVaughan Thomas as he accompanied and documented a flight over Berlin in 1943. The crafting and creating of this experience, and its use, serves to highlight some of the potential benefits of VR across higher education. Students can experience environments that inform their learning goals, but which cannot be accessed in standard learning environments; in this case because the recreation of a WWII raid on Berlin is impossible, but in other cases, due to health and safety, cost or other practical concerns. Taking this application as an example, VR could allow students to occupy a particular space in an embodied manner and actively participate in that space as a form of active learning on WWII.

The potential of $V R$ as a medium in education is grounded in the possibility of this deep embodiment and immersion. A VR headset provides an enclosed visual field for the user; headphones cancel out the sound of the outside world; haptic devices can provide sensory feedback loops of touch, pain, heat or cold. While immersion is a feature of many kinds of effective media used in educational contexts-a student can feel immersed in a good novel, at a compelling play, in the cinema with a good film-the potential and promise of VR is to intensify, perfect and idealise immersion because of the

\section{Correspondence}

Leighton Evans (l.evans@swansea.ac.uk)

doi: 10.1255/vrar2018.ch2

Citation: L. Evans, "Barriers to VR use in HE", in Proceedings of the Virtual and Augmented Reality to Enhance Learning and Teaching in Higher Education Conference 2018, Ed by J. Hudson and R. Kerton. IM Publications Open, Chichester, pp. 3-13 (2019). https://doi.org/10.1255/vrar2018.ch2

\section{(c) 2019 The Author}

This licence permits you to use, share, copy and redistribute the paper in any medium or any format provided that a full citation to the original paper is given.

Print ISBN: 978-1-906715-30-4 Online ISBN: 978-1-906715-28-1 
sensory affordances of the medium. Being immersed in a VR world might just be the most intense media experience we can have-and this leads to immense potential for educational application. Psotka (1995) argued that immersion is the key value added to the educational experience when using VR, and some of the suggested effects of this increase in immersion include increasing the speed of learning, adding depth to the processing of information that aids the retention of information (Freina \& Ott, 2015), and offering new kinds of storytelling experience, like the Berlin Blitz (Powell, Garner, Shapiro, \& Paul, 2017). VR has already made an impact in the fields of training and applied education, with particularly strong impact in medical training (see Riener \& Harders, 2012; McGrath, Taekman, Dev, Danforth, \& Mohan et al., 2017; Westwood, Westwood, \& FelländerTsai, 2016), training in military situations and simulations (see Seidel \& Chatelier, 1997), architectural design training and implementation (Whyte \& Nikolic, 2018) and construction training (Sher, Williams, Gameson, \& Sherratt, 2012). Medical treatments in VR for posttraumatic stress disorder (Reger, Koenen-Woods, Zetocha, Smolenski, Holloway, et al., 2016), eating disorders (Riva, 2017), mental health disorders (Freeman, Reeve, Robinson, Ehlers, Clark, et al., 2017), stroke rehabilitation (Laver, George, Thomas, Deutsch, \& Crotty, 2015) are emerging as examples of uses for $V R$ technology that can provide value to HE through the provision of uniform, simulated training scenarios that are easily applied to teaching and learning in higher education (HE).

For VR to be, or to become, an important aspect of the mix of technologies that augment teaching and learning in HE, it would appear self-evident that VR itself must be: a medium that students can access; that developers can work within and on with assurance that the medium is sustainable and efforts will be used, and that materials, experiences and exercises created in VR will be accessible and not subject to obscurity like educational resources created in virtual environments such as Second Life. At the time of writing, there is still a sense of waiting for $V R$ to take off as a medium that could support such activities (Evans, 2018). that there are considerable barriers to the use of VR in the form available in 2018 (at the time of writing this paper). The focus of this paper is to assess what those barriers are, and whether they can be overcome in a manner that will allow for the potential of immersive educational experiences through VR to be realised in the near future. Understanding the barriers to $V R$ as a concept could be investigated in several ways through empirical research. The research underpinning this paper was a case study into the barriers to the uptake of consumer VR consisting of interviews with 21 designers, programmers and commercial managers. This research fieldwork was conducted between August and December 2017 in Brighton, UK. Brighton was chosen as a site of study due to the community of VR makers, designers, programmers and advocates that had coalesced around the Digital Catapult Immersive Lab (Hills-Duty, 2017).

\section{Methods}

\section{Participants}

17 Brighton-based VR professionals and 3 artists who were performing $V R$-based theatre installations in Brighton were interviewed. 16 male and 4 female professionals were interviewed from a convenience sample of VR professionals associated with the Immersive lab. By profession, these individuals self-identified as: 3 company directors; 2 artistic directors; 1 creative lead; 8 VR 'makers' or designers; 1 innovation manager; 1 development manager; 1 VR programmer; 2 marketing managers of VR companies; and $1 \mathrm{VR}$ narrative designer.

\section{Procedures}

As part of the informed consent granted by participants, the responses reported in this paper have been anonymised, as such, pseudonyms are used below. Semi-structured interviews were used, ranging in length from 20 minutes to 2 hours. The interviews were based around the core conceptual question "what is the immersive potential of VR?", with a subset of questions focussing on the barriers to the use of VR in its current form. The intention of the research was to make available an outline of a series of views of professionals engaged in designing immersive experiences on what barriers they experience in creating VR in practice, and what barriers exist to the uptake of the $V R$ experiences that they create. All of the interviewees have been, or were at the time of interview, involved in the creation of educational experiences in VR. Following a thematic analysis of the material (Joffe \& Thompson, 2011), an inductive approach to analysis where the coding of data occurred without attempting to fit the data into a pre-existing frame or model (thanks to there not being one on barriers to VR to 
draw upon), five repeated barriers to the use of consumer VR were identified that reoccurred across interviews. To address the aims of the present study, those barriers are re-contextualised in terms of educational applications of VR.

\section{Results and discussion \\ Barrier 1: The materiality of VR}

VR is heavily dependent on materiality to achieve the desired virtual effects of embodiment and immersion, but it is the material aspect that currently creates many of the barriers to VR usage. While large and heavy headmounted displays (HMDs) are gradually making way for lighter and more comfortable headsets, this is a process that has not yet met its logical end-point with standalone HMDs that match the technological capabilities of tethered HMDs. Wireless connections are beginning to replace heavy and cumbersome cables that connect HMDs to computers or consoles, but without the processing power of base PCs the visual effect of VR can be compromised. Stand-alone HMDs are allowing for a freedom of movement untethered from stationary computers but recreating the 6 degrees-of-freedom experienced in tethered VR is still in development. The materiality of $V R$ as a tethered technology was identified as a significant barrier to VR use in interviews:

The wires are the things that really bug me. If anything, I feel strangled by the wires; the tethering is something that I really hope goes away very fast. We are building on the assumption that we're not going to need those soon because they just strangle development they strangle testing, they're awful.

(Denise, visual artist)

While makers are planning for a cable-cutting moment in VR (which arguably has arrived with the Oculus Go and HTC Vive Focus and the forthcoming Oculus Quest, although not with the high-end VR HMDs such as the Vive or Rift in 2018), the construction of VR experiences and applications currently are shaped by the physical connection of HMD to the computer or console, and the restrictions that this physical coupling places on movement, gesture and positioning while using VR. Obviously, this will have a shaping effect on the kind of $V R$ environments and experiences that can be designed in an educational context. For example, the inability of current-generation HMDs to allow for pass-through experiences, where the user can see the real world with a VR headset on (Alfredo, 2018), also means that the spaces within which VR can be used within are shaped by a limited affordance for movement or locomotion. Material constraints are not limited to the headsets though. Haptic interfaces are also problematic with regards to barriers to VR use. Haptic interfaces in VR fit into a long history of tactile interfaces between humans and media (Parisi, 2018; Paterson, 2007) and discourses on VR have included notions of the importance of the virtualisation of touch and tactile realism since the early 1990s (Rheingold, 1991: 323). As Parisi (2016: 166) notes, haptic interfaces have existed in a state of "perpetual immanence" across all media, awaited but not quite available. Perfecting haptic interfaces as a means of improving immersion in VR is contingent upon technological advances which, at the time of writing this paper, are not available but which may be available soon-mimicking the wait for untethered VR. The limitations of haptic interfaces mean that the translation of the body and movement is very limited in current VR systems:

Right now, we don't track the whole body. So, we track hands and heads and that allows us to make a semi-accurate estimation of what the player looks like; where they are in the world. I do think you need the full person as they look in real life in that world. Which we can't do right now.

(Osian, designer)

VR has been described as the medium in which interactive biological motion is emphasised (Lanier, 2017) but embodied cognition, the idea that the body plays a role in cognition itself (Merleau-Ponty, 1962) and the critical conceptual element in the use of VR in education, will be difficult when the entire body is not tracked or represented in VR. Current technological fixes for this issue are not seen as being sufficient to address the problems with translating the body:

There's a lot of shortcuts... for instance we could put a tracker on the hand, and we could put trackers on the feet but... you get a disconnect between the real-life presence and the virtual avatar. We need to solve full body avatars that look and react as the players are. (Osian, designer)

The shortcuts and techniques for tracking the body detailed here are ad-hoc additions to the haptic interfaces (controllers) bundled with consumer VR hardware, such as the HTC Vive Tracker or custom-built sensors. These ad-hoc solutions however cannot compensate for 
the difficulties that current haptic sensors have in terms of mapping and representing the body in VR. This creates some specific difficulties for co-presence in $V R$, the phenomenon of being in a VR space with other people, which is a critical aspect of the use of VR in educational contexts. The inability to render the body and bodily movements, gestures and expressions accurately (while avoiding the 'uncanny valley'a; see Mori, 2012; Evans, 2018: 56) in VR currently is a major issue with the materiality of the medium. This is allied with a need to avoid cumbersome equipment that restricts movement, like current tactile rigs and many HMDs. A VR experience does not exist without the user, and that user has a body; we cannot be embodied in a VR experience without being embodied in a physical body that is connected to a VR experience through the HMD and other sensory input devices. Hayles (2004, p. 72) argues that this kind of materiality of media acts as 'a connective tissue joining the physical and mental, the artefact and the user'. In a perfect $V R$ experience, the body would be replicated in $V R$, so our movements are replicated exactly in the virtual. The material aspect of $V R$ therefore has a critical role in translating our bodies into the virtual to establish embodiment in VR. The aim of VR is not to position the user as a disembodied subject inhabiting a virtual environment (Hayles, 2010), but to allow for the body to be reproduced to the extent that the user feels embodied in the virtual environment. This aspect of the materiality of VR is critical to realising deep immersion (Brown \& Cairns, 2004), but the replication of the body in $V R$ requires several material systems.

This is important because the materiality of $V R$ is often overlooked as a factor in the usage and adoption of the medium (Evans, 2018). VR is obviously a medium that is concerned with the virtual-as a basic definition VR generates artificial, computer-generated environments for users to experience. However, VR is also undoubtedly 'material', as accessing VR means having to deal with physical objects and digital systems that are contingent on physical wires, base units and headsets. The head-mounted display, the cables, the connections to computers or the connection to the complex network of

aThe 'uncanny valley' refers to a phenomenon where the closer representations of humans comes to reality in a virtual simulation, the more likely we are to reject that experience as being realistic. In essence, the more realistic the replication of humans, the more the experience deteriorates for the user. wires and servers of the internet mean that VR always has a material aspect as well as a virtual aspect (Evans, 2018). Thus, when considering the use of $V R$, it is implicit that one should consider the physical form of VR that users will need to access a VR environment. This is important because as Miller (2015, p. 68) argues, developments such as VR mean that humans form assemblages of embodied and extended cognition with technologies that 'allow people to experience greater emotional and imaginative relations with media'. Miller's assemblage view contextualises the educational use of $V R$ as a form of extended cognition that relies on physical embodiment as well as experiential exposure to $V R$. VR is a medium that allows students to experience the novel and unobtainable in a learning environment, but this possibility is contingent on the assemblage between student and VR which enables embodied, emotional, extended cognition. The materiality of $V R$-the physical basis and physical realisation of the medium through the apparatus used-is therefore critical in understanding how the deep immersive state desired for learning in HE is established and maintained. For VR to be an accessible and useable medium in education, the morass of cables, wires and tethering to PCs needs cutting - without the quality of experience being cut with the cable. Moreover, the ability to map the body to achieve a level of embodiment is quite distant at this point, and partial embodiment-it could be argued-will only lead to partial immersion and engagement in a VR experience where total immersion is desired.

\section{Barrier 2: Interfaces}

The haptic interfaces for VR are a major issue in the use of the medium at present, but so are the digital interfaces that one encounters when in VR.

Unfortunately, because most of the people who come from a VR space come from traditional gaming or traditional menu-type systems, they try to shoe-in existing menus that exist on screens and computers. Which I don't think lends anything to the experience because you never see a floating wall of buttons in your life. It's intrusive and it automatically means it's not rooting somebody in the experience, it's reminding somebody that it's an invented space and that there's stuff going on outside that space.

(Andy, Director of VR company)

The use of gaming-style menus in VR has a place in the navigation of applications, but by presenting an incon- 
gruous menu that reminds the user that this is VR, not reality, there is an immersion-breaking factor which is also related to a wider point about the development of VR experiences. The dominance of game-style interfaces within VR applications can be contextualised in the dominance of gaming as a cultural form in VR, showing that VR has yet to develop a style of its own away from gaming. This argument about the unsuitability of gaming interfaces echoes that by Jaron Lanier (2017) that VR designers should be fighting against things learned from gaming-even in designing games for VR. The over-reliance on gaming interfaces prevents the development of VR specific interfaces that would act to improve immersion in VR in terms of the experience itself. The adaptation of other media into VR simply as a facsimile of their original form poses significant issues for the use of $V R$ in an educational context as there is an assumed familiarity and habituation to this kind of menu and navigation system from game players. However, in using VR in an educational context there needs to be an appreciation that the audience for the experience may not be gamers, or have any experience of gaming; indeed, it could be argued that a design from gaming principles either consciously or unconsciously prejudices against users that are not gamers. As a VR experience in an educational context will often be intended for use with people who are not familiar with these kinds of interface, there emerges a significant design issue for educational VR. While game navigation may be parsimonious and familiar to designers, it does not logically follow that the same will be true for users-and having a design that is difficult to use would be a significant barrier to use and immersion in VR.

\section{Barrier 3: VR sickness}

One commonly cited barrier to using VR is virtual reality sickness, a phenomenon like motion sickness but without any need for motion. Brooks, Goodenough, Crisler, Klein and Alley et al. (2010) argue that virtual reality sickness may be a major barrier to the use of $V R$, and the phenomenon has been termed 'cybersickness' (Lawson, 2014, p. 531) with a range of symptoms such as general discomfort, headaches, drowsiness and disorientation. Even in experienced VR users such as the developers and makers interviewed for this research, VR sickness is an uncomfortable phenomenon:

So for instance I notice that when I spend too long on VR there are a bunch of effects right so one is just physical like my face feels different because l've had the headset on, I see the world differently cause I'm used to looking in the different way it's almost like when you've got the magic mirror book thing, you have to use your eyes differently, so its reconfiguring my brain to look differently so I then subsequently see the world a bit differently which is not a bad thing because I see the world in an interesting new way, my relationship to objects is different. There's almost like a hangover thing for VR and suspect that that's normal and we just have to evolve as humans if we're going to have VR in our lives.

(Denise, visual artist)

There is a critically important point raised here when considering the use of VR in an educational context. With regards to the nausea mentioned above, evidence suggests that women are more susceptible to VR sickness than men (Munafo, Diedrick, \& Stoffregen, 2016). There are a range of possible explanations offered for this gender disparity including: women having a wider field of view than men (Park, Allen, Fiorentino, Rosenthal, \& Cook, 2006), which suggests that VR headsets and lenses in HMDs show a bias towards men at the design stage; hormonal differences between men and women (Kennedy, Lilienthal, Berbaum, Baltzley, \& McCauley, 1989) or gender differences in depth cue perception due to men favouring motion parallax (which is prioritized in VR) and women favouring shape-from-shading (Biocca, 1992; boyd, 2001, 2014). The idea that female users are more susceptible to VR sickness asks questions about the design and testing of VR systems in general, and whether these systems are being designed for all, or only for the 'most likely' to buy VR technology in the view of producers. If economic rationality is guiding design principles that disadvantage female VR users, then this barrier to VR use is coded into the design of systems and is therefore hugely problematic. In an educational context, this is even more worrying-a technology that, by design, excludes or disadvantages female students with regards to experiences raises serious questions regarding equality of provision.

\section{Barrier 4: Cost}

It is very much chicken and egg - if there is a bigger market place then it can support more content but you cannot get more content without a bigger market place. Or rather you cannot get the funding to make more content. Or a lower risk opportunity 
to at least break even but it is about patience and everyone talks about having to have patience with VR so early investor overhype-sorry, early analyst overhype and media overhype, the three contributing factors they all then turned around and at the end of 2016 proclaimed commercial VR when at the beginning they were saying, there was going to billions and then by end saying, oh it was not billions, it is a failure. But if you look at Facebook and Oculus they have been saying it is a ten-year game-it is a long game.

(James, Director of Immersive Technologies)

James outlines the cost barrier to VR clearly. Highquality content is needed to push VR as a medium (of course, including educational content) but with a relatively small market the likelihood of wide-scale investment in VR content is low. This means that the development of content, including educational content, for $V R$ is being limited by the economic limitations of the medium at present. In an educational context this may mean that institutions do not only have an equipment cost to bear, but also a development cost as VR experiences must be developed in house as the market (and design-side makers and developers) expands.

Cost is a direct barrier to VR; unlike VR sickness, which requires trying $V R$ to experience discomfort, the cost of $V R$ systems will prevent people from engaging with VR at any level. While there are affordable VR systems, such as smartphone-based mobile VR platforms such as Google Cardboard, these solutions still require an expensive smartphone being repositioned as a VR system. The launch of stand-alone VR HMDs such as the Oculus Go, HTC Vive Focus and Lenovo Mirage in 2018 offer consumer VR systems for between $£ 200-£ 400$, but in a crowded market for consumer electronic spending this still represents an investment that many may not be prepared to make without a clear and obvious need for VR. In an educational context, the institution may be expected to meet the costs of $V R$, but this then limits the medium to a campus-based technology, arguably limiting the scope of VR in higher education. The price of consumer $V R$ was explicitly linked to the economic viability of the VR industry as a whole in the research, with respondents clear that without an economic base for consumer VR, costs are unlikely to decrease.

I see the next five, six years. Facebook already made a huge investment in it and it's dropped the price down for the Oculus. So, I imagine the next five years will have prices coming down to more reasonable point, maybe like eighty quid [pounds], fifty quid for $a$ VR headset. Like a stand-alone VR kit. And then you'll have more developers going for it because more people will have the technology assisting it for revenue on return investment.

(Osian, designer)

The 'Facebook factor' in the cost of VR is a significant issue even as their presence may reduce the cost barrier to VR. For VR to develop into a viable medium, cost reduction needs to be mirrored by an increase in content development which would make VR an attractive proposition. Long-term investment by major organisations should make this possible but raises significant issues for educational use. If the affordable end of the VR market is dominated by Facebook-owned (and increasingly controlled, Evans, 2018) Oculus, then many questions emerge about the suitability of having a mass dataharvesting and data-selling organisation, mired in political controversy, with a major role as a platform-provider in higher education. The barrier of cost may eventually see another barrier emerge if it is overcome-an ethical barrier concerning the use of data produced in VR and who controls the use of that data.

\section{Barrier 5: The language of VR}

Exactly, cause it's a language we need to develop a whole new language, yeah and most of us don't know what that is and technical difficulties are still quite substantial due to this.

(Leon, Artist and VR designer)

The language of $V R$ is a concept that needs a great deal of unpacking in the context of educational use but was one that was frequently mentioned by $V R$ professionals in many different contexts. This barrier to $V R$ is concerned with a conceptual gap between the expectations of VR and the ability to articulate VR experiences to users and customers by VR developers. In a nutshell, there is a disconnect between what VR makers are talking about and trying to articulate and what client-side consumers are asking for and receiving in VR. This lack of fit between the discourses of VR creators and consumers can be attributed to the lack of an overarching discourse or language of VR (Evans, 2018); there is not yet the common knowledge of $V R$ techniques or capabilities that allows for the vision customers want to be articulated in a way that can elicit a response from makers. Conversely, VR makers are promoting and selling VR products without 
a set of terms that can articulate the effort and skills needed for effective VR 'making'.

The lack of a language of $V R$ or discourses emerging from VR means that conceptual understanding of the medium often comes from discourses on other, tangential media such as gaming. Due to this, there is some indication that people are convinced VR is an extension of the games industry rather than a medium that offers a myriad of experiences (including education):

But one of the key failures I would say over the past few years has been the overemphasis on the gaming market so if you look at 2014 onwards it has all been games, games, games and nothing else. And the mainstream press on board, all the platforms pushing their gaming elements but ultimately that has not been hugely successful.

(James, Director of Immersive Technologies)

The adoption of gaming discourses might have been inevitable as gaming and the cultures around gaming provide a language to contextualise and discuss VR in a way that makes sense. As Lanier (2017) argues, the cause of the language problem in VR is that people are using language tools borrowed from other mediums or technologies to describe VR. The problem, for Lanier, is that there is not yet a language of VR and that VR is in its own terms a language (Lanier, 2017: 222) that has not yet been 'translated'. Due to the lack of a language of $V R$ that can communicate the conceptual aspects of the medium clearly between developer and those commissioning VR experiences, the experiences of VR are being translated into other language codes such as gaming to be articulated to an audience-with the by-product that VR is considered an extension of gaming. What is needed is a new set of discursive terms for media, such as those discussed by Manovich (2001) in relation to new media. Manovich presented a new language of terms and discourses that allowed for the articulation of key processes and cultural aspects of new media, and these terms became part of a common parlance on new media that allowed for communication of concepts and uses of new technology. There is not, yet, the same language of $V R$. This means that the discussion of the experiences made for VR are grounded in other media discourses which misrepresent the medium. Furthermore, communication about the benefits (especially educational) is difficult without resorting to familiar language on gaming or other media which does not represent or articulate the benefits of VR clearly.The difficulty in translating VR is not just an inability of understanding on the part of those outside VR that must be convinced of the benefits of VR. People working in the VR community also struggle to articulate VR in a language that can be used, and the technological aspects of VR may be a cause of this difficulty in clearly articulating on VR:

I realised that when I talked about gaze interaction there was a lot of confusion just in that area that people do not understand that there is different type of gaze interactions and then as an academic I was like, there needs to be a discourse, a language created around that.

(Rob, VR creator and academic)

Rob both designs in VR for education and writes about $V R$ in an academic context, and while his work articulates the technological concepts of VR well to an academic audience that is not a form of discourse that will necessarily translate well to a non-academic audience. The need for a language to express these concepts to people outside of VR and within VR communities is essential in overcoming this discursive barrier. Communicating to people outside the VR community was far more important than intra-VR community communications for other interviewees though:

Do you think the people who commission you have any idea of what goes into making a genuinely immersive experience? They have a lack of knowledge. There's a lot of work there that needs to be done prior to doing pitches. And it's very sort of simple things that to us in hindsight, looking back on, we have taken for granted.

(Nick, UX and Sales manager for VR Company)

Nick's role is to pitch for contracts with companies (which have included Universities) that have expressed interest in promoting their businesses with VR experiences. While he converses within his own business and with colleagues in VR who are technologically informed, the articulation of $V R$-specific terms to customers outside the community is difficult. The act of selling VR to sometimes sceptical potential clients lacks a mutually coherent frame which would make the technical and artistic benefits of VR more readily apparent. In the context of educational VR this is important-if the makers of educational VR cannot articulate the benefits to and for educators or students, it is unlikely that the medium will be used.

People cannot say what it is and that is confusing people because I think there is a key thing of confusion with the terminology coming from marketing 
companies. We need to create artworks that communicate the terminology used and have the capacity to inform practitioners across the board on these processes. I feel like that is part of what we would call the meta language of this new media.

(Rob, VR creator and academic)

The need for a language of $V R$ is therefore critical in communicating the potential of VR accurately. The need for a new language of $V R$ is also acute in respect of communicating between the audience and VR makers: In VR, we now have a gap between creator of contentgiving a message the audience can decode, because there is very little shared language now and very little understanding from the creator's point of view as to what the audience can take and understand. And the unfortunate thing I think about VR now is that too many people are taking a medium such as a PC game, throwing it into this new space and expecting it to work, and people don't know how to consume. People don't like it-and because they don't like it, they don't buy it.

(Andy, Director of VR company)

The lack of a language of $V R$ creates a distance between VR makers and an audience, and this gap manifests itself in VR experiences that are not readily or easily understood by audiences-obviously, a major issue for educational VR in creating an understanding of environments for learners. VR as a medium involves far more explanation than other mediums because of what $V R$ requires in terms of designing an experience for an end-user and how that experience is developed:

I think that people need to understand that compared to other mediums like video and photography and print... you create that content. For us, because we control the person's environment, absolutely everything they see and experience that means you need to go through a load of different hoops and hurdles to make sure they have that experience. It takes a lot longer, there are certain hoops you need to jump through and I think that's why not many people realise we are controlling the environment and the users around it. We need to create that in the correct way or people aren't going to enjoy it. As you say, otherwise you don't get that 'magic'...

(Nick, UX and Sales manager for VR Company)

Entertainment is not the primary focus of VR in an educational context (learning and development being more fundamental), but the making of effective VR expe- riences for educational clients does involve more than only making a VR experience-it also involves explaining how engagement and control are far greater factors in a VR experience than in other media (even in the age of social media). To create something that has the 'magic', the language barrier to VR must be overcome with careful translation and explanation to educate as well as produce. James outlines the process that his company uses to overcome the language barrier of $V R$ :

We have what we call a 4D approach. So, we have: discover, design, develop, deploy. So typically, people would come to us saying, we have heard about VR-what is it. We will then run through a half day of educational demos, training, tutorial, giving them a one-to-one VR so they can understand how it could potentially benefit them. And then we work with them to outline a specific goal or objective or point they are trying to address or meet. And then we will co-design, collaborate with them, with their key stakeholders to design whatever it is. Typically alongside development training, on boarding, many of the corporates are really aware that people growing up with smartphones and tablets and much more interactive media these days, they are bored and won't engage with your standard computer based training-click through to the next screen and here's more words about whatever it is you're doing or what you're trying to learn, so let's drop down from the list to finish what we are learning. But also putting learning by doing into practice. So that people can train safely, fail safely, but also build up muscle memory in terms of being engaged with their real-world counterparts.

(James, Director of Immersive Technologies)

James' company makes health and safety training VR applications and educational VR. The process of translation and negotiation that they perform will be tailored to each client, but the principle of the 4D approach is uniform, as well a major drain on time and resources. This approach appears necessary though in the infancy of educational and training VR as a medium. Building $V R$ experiences requires a careful process of tutorials and education on VR, and so VR for education is not just about teaching students but also teaching educational professionals about the medium. The implications of this approach are clear: given the difficulty in articulating the form, content and uniqueness of VR extra education is needed to overcome this barrier. Turning to other linguistic codes and discourses from other media 
to explain the medium is in itself a barrier to the understanding of the principles and benefits of educational VR and does not solve the problem at hand.

\section{Conclusion}

The barriers to educational VR identified in this research are by no means insurmountable. Overcoming these barriers in nearly every case involves investment: investing in technology to solve hardware issues, which has already been seen in the consumer sphere with the Oculus Go; investing in development and content to improve interfaces; investing in research to solve VR sickness issues (especially regarding female users); and investing in reducing the cost of consumer VR systems. However, without a return on investment through learning outcomes and positive student evaluations of the medium there is unlikely to be the kind of investment identified here to resolve these barriers in the shortterm (Evans, 2018). A long-term approach to VR use in education may lead to the phasing in of research, development and content creation that will see these barriers overcome, but this may be over several years. For now, this means that the medium may develop slowly and gradually as an educational tool rather than having an immediate impact that changes the face of education altogether. This, of course, is not a negative-a reflective, research-driven approach to the implementation and refinement of VR in educational contexts will undoubtedly be beneficial in terms of learning and development.

More critically, investment will not necessarily solve the barrier of having a language of VR to communicate concepts critical to the medium within the educational space. Unless there is considerably more cultural impact than the medium has at present, a popular discourse of $V R$ that conveys and accurately communicates the possibilities, affordances and benefits of VR is unlikely to develop in the short-term. This lack of a language to 'talk VR' means that concepts in VR are continually grounded in other media-asking the question of why not just stick to that media instead of VR? - and that VR itself is not seen as revolutionary, despite the possibilities of deep immersion, embodiment and presence that the medium offers. This can only be solved with increasing the points of discourse between VR designers and users through usage. Educational use of $V R$ may well be a major contributor for the development of a language of VR as in an educational context more people could be directed towards VR as a medium and have experiences that are linked to goals extrinsic to the VR experience itself, bringing a context with benefits of $V R$ to the user. However, without a grounded understanding of the possibilities, limitations and potential applications for VR in the first instance, popularising VR in higher (or any) education presents a distinct and difficult barrier.

\section{Closing comments}

VR as a consumer medium is in its very infancy, and although the medium has a long history in training and education contexts dating back to Sutherland's (1968) Sword of Damocles headset for trainee pilots there is still a huge amount of development to occur in this field. The barriers identified in this research are likely to fall one by one as the medium becomes a part of the everyday digital media milieu, and in time this paper may seem a relic. However, in the short-term each barrier presents specific issues that need to be overcome if the full potential of VR is to be realised in education.

A potential solution to some of these barriers comes from the 4D approach advocated by the interviewee 'James' in this research. That approach involves VR makers themselves setting out to discover, design, develop and deploy with regards to educational VR applications. This approach advocates a partial use of knowledge transfer procedures where VR makers are tasked not just with making VR experiences but also engaging with educational providers and users to understand their needs and bring that to the design process. The design part of this approach is, when unpacked, a long process that can deal with many of the barriers to VR including the issue of a language of VR. As a model of VR development, the 4D approach could help the use of $V R$ in education through inclusivity and knowledge transfer, and therefore is proposed here as a potential model of best practice (although further research on the efficacy and effectiveness of this method is required) for developing VR- including in education- applications with the aim of avoiding the barriers identified in this paper. In taking this approach, the barriers of could be countered through careful planning, execution and collaboration across the entirety of a VR project (idea, design, creation, testing, implementation and evaluation)-but this is a skill in itself. 
Undoubtedly, further research is required on how these barriers to the use of VR affect individual users and how they manifest in educational contexts for student users also needs extensive examination. Until the day comes when students are fully accustomed to using a VR headset as part of their education, these barriers and others that will emerge over time require research and acknowledgment if the full added value of VR through immersion is to be used to improve educational outcomes.

\section{References}

Alfredo. (2018, April 01). The case for passthrough with VR. Retrieved 03 April 2018 from https://medium. com/@alfredos/the-case-for-passthrough-with-vra620b26e42bb

BBC (2018). New BBC VR film flies you to Berlin at the height of the Second World War. Retrieved 19 December 2018 from https://www. bbc.co.uk/mediacentre/latestnews/2018/berlinblitz-vr

Biocca, F. (1992). Will simulation sickness slow down the diffusion of virtual environment technology? Presence: Teleoperators and Virtual Environments, 1(3), 334-343. https://doi.org/10.1162/ pres.1992.1.3.334

Boyd, D. (2001). Depth cues in virtual reality and the real world: Understanding differences in depth perception by studying shape-from-shading and motion parallax. (Unpublished undergraduate dissertation). Brown University.

Boyd, D. (2014, April 3). Is the Oculus Rift sexist? (plus response to criticism). Retrieved February 1, 2018, from http://www.zephoria.org/thoughts/ archives/2014/04/03/is-the-oculus-rift-sexist.html

Brooks, J.O., Goodenough, R.R., Crisler, M.C., Klein, N.D., Alley, R.L., Koon, B.L., Wills, R.F. (2010). Simulator sickness during driving simulation studies. Accident Analysis \& Prevention, 42(3), 788-796. https://doi.org/10.1016/j.aap.2009.04.013

Brown, E., \& Cairns, P. (2004). A grounded investigation of game immersion. Extended Abstracts of the 2004 Conference on Human Factors and Computing Systems- CHI 04. https://doi. org/10.1145/985921.986048

Evans, L. (2018). The re-emergence of Virtual Reality. London: Routledge.
Freeman, D., Reeve, S., Robinson, A., Ehlers, A., Clark, D., Spanlang, B., \& Slater, M. (2017). Virtual reality in the assessment, understanding, and treatment of mental health disorders. Psychological Medicine, 47(14), 2393-2400. https://doi.org/10.1017/ s003329171700040x

Freina, L., \& Ott, M. (2015). A literature review on immersive virtual reality in education: state of the art and perspectives. Retrieved 19 December 2018 from https://www. researchgate.net/publication/280566372_A_ Literature_Review_on_Immersive_Virtual_Reality_in_ Education_State_Of_The_Art_and_Perspectives

Hayles, N.K. (2004). Print is flat, code is deep: the importance of media-specific analysis. Poetics Today, 25(1), 67-90. https://doi.org/10.1215/0333537225-1-67

Hayles, N.K. (2010). How we became posthuman: Virtual bodies in cybernetics, literature and informatics. Chicago: Univ. of Chicago Press.

Hills-Duty, R. (2017, October 04). Digital catapult announce new immersive lab in Brighton. Retrieved April 04, 2018, from https://www.vrfocus. com/2017/10/digital-catapult-announce-newimmersive-lab-in-brighton/

Joffe, H., \& Thompson, A.R. (2011). Thematic analysis. In D. Harper (Ed.), Qualitative Research Methods in Mental Health and Psychotherapy Qualitative Research Methods in Mental Health and Psychotherapy: A Guide for Students and Practitioners (pp. 210-223). New York: Wiley.

Kennedy, R.S., Lilienthal, M.G., Berbaum, K.S., Baltzley, D.R., \& McCauley, M.E. (1989). Simulator sickness in U. S. Navy flight simulators. Aviation, Space, and Environmental Medicine, 60, 10-16.

Lanier, J. (2017). Dawn of the New Everything: A Journey through Virtual Reality. London: Bodley Head.

Laver, K.E., George, S., Thomas, S., Deutsch, J.E., \& Crotty, M. (2015). Virtual reality for stroke rehabilitation. Cochrane Database of Systematic Reviews, 12(2), CD008349. https://doi.org/10.1002/14651858. cd008349.pub3

Lawson, B.D. (2014). Motion sickness symptomatology and origins. In K.S. Hale \& K.M. Stanney (Eds.), Handbook of Virtual Environments: Design, Implementation, and Applications (pp. 531-599). Boca Raton, FL: CRC Press. 
Manovich, L. (2001). The Language of New Media. Cambridge, MA: MIT Press.

McGrath, J.L., Taekman, J.M., Dev, P., Danforth, D.R., Mohan, D., et al. (2017). Using virtual reality simulation environments to assess competence for emergency medicine learners. Academic Emergency Medicine, 25(2), 186-195. https://doi.org/10.1111/ acem.13308

Merleau-Ponty, M. (1962). Phenomenology of Perception. London: Routledge.

Miller, J. (2015). The dematerializing interface. Westminster Papers in Culture and Communication, 10(1), 66-80. https://doi.org/10.16997/wpcc.213

Mori, M. (2012). The uncanny valley [From the Field] (K. Macdorman \& N. Kageki, Trans.). IEEE Robotics \& Automation Magazine, 19(2), 98-100. https://doi. org/10.1109/mra.2012.2192811

Munafo, J., Diedrick, M., \& Stoffregen, T. A. (2016). The virtual reality head-mounted display Oculus Rift induces motion sickness and is sexist in its effects. Experimental Brain Research, 235(3), 889-901. https://doi.org/10.1007/s00221-016-4846-7

Parisi, D. (2016). Eine technik der Medienberührung: Kinästhetische Displays und die Suche nach Computerhaptik. Maske Und Kothurn, 62(2-3). https://doi.org/10.7767/muk-2016-2-310

Parisi, D. (2018). Archaeologies of touch: Interfacing with haptics from electricity to computing. Minneapolis: University of Minneapolis Press.

Park, G.D., Allen, R.W., Fiorentino, D., Rosenthal, T.J., \& Cook, M. L. (2006). Simulator sickness scores according to symptom susceptibility, age, and gender for an older driver assessment study. PsycEXTRA Dataset. https://doi.org/10.1037/e577802012-007

Paterson, M. (2007). The senses of touch: Haptics, affects and technologies. Oxford: Berg.

Powell, W., Garner, T.A., Shapiro, S., \& Paul, B. (2017). Virtual reality in entertainment: The state of the industry. Retrieved 19 December 2018 from http:// www.bafta.org/sites/default/files/uploads/vrpaperbaftasept2017.pdf

Psotka, J. (1995). Immersive training systems: Virtual reality and education and training. Instructional Science, 23(5-6), 405-431.

Reger, G.M., Koenen-Woods, P., Zetocha, K., Smolenski, D.J., Holloway, K.M., Rothbaum, B.O. \& Gahm, G.A. (2016). Randomized controlled trial of prolonged exposure using imaginal exposure vs. virtual reality exposure in active duty soldiers with deployment-related posttraumatic stress disorder (PTSD). Journal of Consulting and Clinical Psychology, 84(11), 946-959. https://doi.org/10.1037/ ccp0000134

Rheingold, H. (1991). Virtual reality. New York, NY: Touchstone.

Riener, R., \& Harders, M. (2012). Virtual reality in medicine. London: Springer.

Riva, G. (2017). Letter to the editor: Virtual reality in the treatment of eating and weight disorders. Psychological Medicine, 47(14), 2567-2568. https:// doi.org/10.1017/s0033291717001441

Seidel, R.J., \& Chatelier, P.R. (1997). Virtual reality, training's future?: Perspectives on virtual reality and related emerging technologies. New York: Plenum.

Sher, W., Williams, A., Gameson, R., \& Sherratt, S. (2012). Changing skills in changing environments: skills needed in virtual construction teams. In C.S. Lanyi (Ed.), Applications of Virtual Reality (pp. 31-49). INTECH Open Access Publisher.

Westwood, J.D., Westwood, S.W., \& Felländer-Tsai, L. (2016). Medicine meets virtual reality 22: NextMed / MMVR22. Amsterdam: IOS Press.

Whyte, J., \& Nikolic, D. (2018). Virtual reality and the built environment. London: Routledge. 